\title{
On Right Caputo Fractional Ostrowski Inequalities Involving Three Functions
}

\author{
Deepak B. Pachpatte \\ Department of Mathematics, Dr. B.A.M. University, Aurangabad, Maharashtra 431004, India
}

Correspondence should be addressed to Deepak B. Pachpatte; pachpatte@gmail.com

Received 28 August 2012; Accepted 27 December 2012

Academic Editor: Abdallah El Hamidi

Copyright (C) 2013 Deepak B. Pachpatte. This is an open access article distributed under the Creative Commons Attribution License, which permits unrestricted use, distribution, and reproduction in any medium, provided the original work is properly cited.

We establish Ostrowski inequalities involving three functions in right Caputo fractional derivative in $L_{p}$ spaces.

\section{Introduction}

In 1938, Ostrowski proved the following useful inequality.

Let $f:[a, b] \rightarrow R$ be continuous on $[a, b]$ and differentiable on $(a, b)$ whose derivative $f^{\prime}:[a, b] \rightarrow R$ is bounded on $(a, b)$, that is, $\left\|f^{\prime}\right\|_{\infty}=\sup _{t \in(a, b)}\left|f^{\prime}(t)\right|<\infty$. Then

$$
\left|f(x)-\frac{1}{b-a} \int_{a}^{b} f(t) d t\right| \leq\left[\frac{1}{4}+\frac{\left(x-\frac{a+b}{2}\right)}{(b-a)^{2}}\right]\left\|f^{\prime}\right\|_{\infty},
$$

for any $x \in[a, b]$. The constant $1 / 4$ is best possible.

In $[1,2]$ Pachpatte has proved Ostrowski inequality in three independent variables.

In past few years many authors have obtained various generalisation and variant of the above type of inequality and other on fractional as well as time scale calculus see [3-6].

Here we give some basic definition from fractional calculus used in [7-9].

Definition 1. Let $f \in L_{1}[a, b], \alpha>0$. The right and left Riemann-Liouville integrals $I_{a+}^{\alpha} f(x)$ and $I_{b-}^{\alpha} f(x)$ of order $\alpha>0$ with $a \geq 0$ are defined by

$$
I_{a+}^{\alpha} f(x)=\frac{1}{\Gamma(\alpha)} \int_{a}^{x}(x-t)^{(\alpha-1)} f(t) d t, \quad x>a,
$$

$$
I_{b-}^{\alpha} f(x)=\frac{1}{\Gamma(\alpha)} \int_{x}^{b}(t-x)^{(\alpha-1)} f(t) d t, \quad x<b,
$$

respectively, where $\Gamma(\alpha)=\int_{0}^{\infty} e^{-t} t^{\alpha-1} d t$ and $I_{a+}^{0} f(x)=$ $I_{b-}^{0} f(x)=f(x)$.

Definition 2 (see [10, page 2]). Let $f \in A C^{m}([a, b])\left(f^{(m-1)}\right.$ be in $A C([a, b)), m \in N, m=[\alpha], \alpha>0(|\cdot|$ the ceiling of the number). We define the right Caputo fractional derivative of order $\alpha>0$ by

$$
D_{b-}^{\alpha} f(x)=\frac{(-1)^{m}}{\Gamma(m-\alpha)} \int_{x}^{b}(t-x)^{m-\alpha-1} f^{(m)}(t) d t, \quad x \leq b .
$$

If $\alpha=m \in N$, then

$$
D_{b-}^{m} f(x)=(-1)^{m} f^{(m)}(x), \quad \forall x \in[a, b] .
$$

If $x>b$, we define $D_{b-}^{m} f(x)=0$.

Definition 3 (see [9, page 74]). Let $f, g \in A C^{m}([a, b]), \alpha>0$ with $a \geq 0$, and then the fractional integral $I_{b-}^{\alpha} f(x) g(x)$ is defined by

$$
I_{b-}^{\alpha} f(x) g(x)=\frac{1}{\Gamma(\alpha)} \int_{x}^{b}(t-x)^{(\alpha-1)} f(t) g(t) d t, \quad x<b .
$$

We give here the theorems proved in [10]. 
Theorem 4. Let $\alpha>0, m=[\alpha], f \in A C^{m}([a, b])$. Assume that $f^{k}(b)=0, k=1, \ldots, m-1$ and $D_{b-}^{a} f \in L_{\infty}([a, b])$. Then

$$
\left|\frac{1}{b-a} \int_{a}^{b} f(x) d x-f(b)\right| \leq \frac{\left\|D_{b-}^{\alpha} f\right\|_{\infty,[a, b]}}{\Gamma(\alpha+2)}(b-a)^{\alpha} .
$$

Theorem 5. Let $\alpha>0, m=[\alpha], f \in A C^{m}([a, b])$. Assume that $f^{k}(b)=0, k=1, \ldots, m-1$ and $D_{b-}^{a} f \in L_{1}[a, b]$. Then

$$
\left|\frac{1}{b-a} \int_{a}^{b} f(x) d x-f(b)\right| \leq \frac{\left\|D_{b-}^{\alpha} f\right\|_{L_{1}([a, b])}}{\Gamma(\alpha+1)}(b-a)^{\alpha-1} .
$$

Theorem 6. Let $p, q>1 ; 1 / p+1 / q=1, \alpha>1-1 / p, m=[\alpha]$, $f \in A C^{m}([a, b])$. Assume that $f^{k}(b)=0, k=1, \ldots, m-1$, and $D_{b-}^{\alpha} f, h \in L_{q}[a, b]$. Then

$$
\begin{aligned}
& \left|\frac{1}{b-a} \int_{a}^{b} f(x) d x-f(b)\right| \\
& \quad \leq \frac{\left\|D_{b-}^{\alpha} f\right\|_{L_{q}([a, b])}}{\Gamma(\alpha)(p(\alpha-1)+1)^{1 / p}(\alpha+1 / p)}(b-a)^{\alpha-1+1 / p} .
\end{aligned}
$$

\section{Main Results}

Our main results are given in the following theorems.

Theorem 7. Let $\alpha>0, m=[\alpha], f, g, h \in A C^{m}([a, b])$. Assume that $f^{k}(b)=g^{k}(b)=h^{k}(b)=0, k=1, \ldots, m-1$ and $D_{b-}^{a} f, D_{b-}^{a} g, D_{b-}^{a} h \in L_{\infty}([a, b])$. Then

$$
\begin{aligned}
& \mid 3 \int_{a}^{b} f(x) g(x) h(x) d x \\
& -\int_{a}^{b}[f(b) g(x) h(x)+f(x) g(b) h(x) \\
& \quad+f(x) g(x) h(b)] d x \mid \\
& \leq\left\|D_{b-}^{\alpha} f\right\|_{\infty} I_{\alpha}^{\alpha+1}|g(b) h(b)|+\left\|D_{b-}^{\alpha} g\right\|_{\infty} I_{\alpha}^{\alpha+1}|f(b) h(b)| \\
& \quad+\left\|D_{b-}^{\alpha} h\right\|_{\infty} I_{\alpha}^{\alpha+1}|f(b) g(b)| .
\end{aligned}
$$

Proof. Let $x \in[a, b]$ we have

$$
\begin{aligned}
& f(x)-f(b)=\frac{1}{\gamma \alpha} \int_{x}^{b}(t-x)^{(\alpha-1)} D_{b-}^{\alpha} f(t) d t, \\
& g(x)-g(b)=\frac{1}{\gamma \alpha} \int_{x}^{b}(t-x)^{(\alpha-1)} D_{b-}^{\alpha} g(t) d t, \\
& h(x)-h(b)=\frac{1}{\gamma \alpha} \int_{x}^{b}(t-x)^{(\alpha-1)} D_{b-}^{\alpha} h(t) d t .
\end{aligned}
$$

Multiplying (10), (11), and (12) by $g(x) h(x), h(x) f(x)$, and $f(x) g(x)$, respectively, and adding them, we have

$$
\begin{aligned}
3 f(x) & g(x) h(x)-f(b) g(x) h(x) \\
& -f(x) g(b) h(x)-f(x) g(x) h(b) \\
= & \frac{g(x) h(x)}{\Gamma(\gamma)} \int_{x}^{b}(t-x)^{\alpha-1} D_{b-}^{\alpha} f(t) d t \\
& +\frac{h(x) f(x)}{\Gamma(\gamma)} \int_{x}^{b}(t-x)^{\alpha-1} D_{b-}^{\alpha} g(t) d t \\
& +\frac{f(x) g(x)}{\Gamma(\gamma)} \int_{x}^{b}(t-x)^{\alpha-1} D_{b-}^{\alpha} h(t) d t .
\end{aligned}
$$

Integrating both sides of (13) with respect to $x$ and rewriting above equation we have

$$
\begin{aligned}
3 \int_{a}^{b} f(x) & g(x) h(x) d x \\
- & \int_{a}^{b}[f(b) g(x) h(x)+f(x) g(b) h(x) \\
& \quad+f(x) g(x) h(b)] d x \\
= & \int_{a}^{b} \frac{g(x) h(x)}{\Gamma(\gamma)}\left(\int_{x}^{b}(t-x)^{\alpha-1} D_{b-}^{\alpha} f(t) d t\right) \\
& +\int_{a}^{b} \frac{h(x) f(x)}{\Gamma(\gamma)}\left(\int_{x}^{b}(t-x)^{\alpha-1} D_{b-}^{\alpha} g(t) d t\right) \\
& +\int_{a}^{b} \frac{f(x) g(x)}{\Gamma(\gamma)}\left(\int_{x}^{b}(t-x)^{\alpha-1} D_{b-}^{\alpha} h(t) d t\right) .
\end{aligned}
$$

From (14) and using the properties of modulus we have

$$
\begin{aligned}
& \mid 3 \int_{a}^{b} f(x) g(x) h(x) d x \\
& -\int_{a}^{b}[f(b) g(x) h(x)+f(x) g(b) h(x) \\
& \quad+f(x) g(x) h(b)] d x \mid \\
& =\int_{a}^{b} \frac{|g(x) h(x)|}{\Gamma(\gamma)}\left(\int_{x}^{b}(t-x)^{\alpha-1}\left|D_{b-}^{\alpha} f(t) d t\right|\right) \\
& \quad+\int_{a}^{b} \frac{|h(x) f(x)|}{\Gamma(\gamma)}\left(\int_{x}^{b}(t-x)^{\alpha-1}\left|D_{b-}^{\alpha} g(t) d t\right|\right) \\
& \quad+\int_{a}^{b} \frac{|f(x) g(x)|}{\Gamma(\gamma)}\left(\int_{x}^{b}(t-x)^{\alpha-1}\left|D_{b-}^{\alpha} h(t) d t\right|\right) .
\end{aligned}
$$


It is easy to observe that

$$
\begin{aligned}
& 3 \int_{a}^{b} f(x) g(x) h(x) d x \\
& -\int_{a}^{b}[f(b) g(x) h(x)+f(x) g(b) h(x) \\
& +f(x) g(x) h(b)] d x \\
& \leq \frac{\left\|D_{b-}^{\alpha} f\right\|_{\infty}}{\gamma \alpha} \int_{a}^{b}|g(x) h(x)|\left(\int_{x}^{b}(t-x)^{\alpha-1} d t\right) d x \\
& +\frac{\left\|D_{b-}^{\alpha} g\right\|_{\infty}}{\gamma \alpha} \int_{a}^{b}|h(x) f(x)|\left(\int_{x}^{b}(t-x)^{\alpha-1} d t\right) d x \\
& +\frac{\left\|D_{b-}^{\alpha} h\right\|_{\infty}}{\gamma \alpha} \int_{a}^{b}|f(x) g(x)|\left(\int_{x}^{b}(t-x)^{\alpha-1} d t\right) d x \\
& =\frac{\left\|D_{b-}^{\alpha} f\right\|_{\infty}}{\Gamma(\alpha+1)} \int_{a}^{b}(b-x)^{\alpha}|g(x) h(x)| d x \\
& +\frac{\left\|D_{b-}^{\alpha} g\right\|_{\infty}}{\Gamma(\alpha+1)} \int_{a}^{b}(b-x)^{\alpha}|h(x) f(x)| d x \\
& +\frac{\left\|D_{b-}^{\alpha} h\right\|_{\infty}}{\Gamma(\alpha+1)} \int_{a}^{b}(b-x)^{\alpha}|f(x) g(x)| d x \\
& =\left\|D_{b-}^{\alpha} f\right\|_{\infty} I_{a+}^{\alpha+1}|g(b) h(b)|+\left\|D_{b-}^{\alpha} g\right\|_{\infty} I_{a+}^{\alpha+1}|f(b) h(b)| \\
& +\left\|D_{b-}^{\alpha} h\right\|_{\infty} I_{a+}^{\alpha+1}|f(b) g(b)| .
\end{aligned}
$$

The proof of the theorem is complete.

Remark 8. If we take $h(t)=1, g(t)=1$ and hence $\left\|D_{b-}^{a} h\right\|_{\infty}=$ $0,\left\|D_{b-}^{a} g\right\|_{\infty}=0$ in Theorem 7 , then we get Theorem 4 .

Theorem 9. Let $\alpha \geq 0, m=[\alpha], f, g, h \in A C^{m}([a, b])$. Assume that $f^{k}(b)=g^{k}(b)=h^{k}(b)=0, k=1, \ldots, m-1$, and $D_{b-}^{\alpha} f, D_{b-}^{\alpha} g, D_{b-}^{\alpha} h \in L_{1}[a, b]$. Then

$$
\begin{aligned}
& \mid 3 \int_{a}^{b} f(x) g(x) h(x) d x \\
& -\int_{a}^{b}[f(b) g(x) h(x)+f(x) g(b) h(x) \\
& \quad+f(x) g(x) h(b)] d x \mid \\
& \leq\left\|D_{b-}^{\alpha} f\right\|_{L_{1}([a, b])} I_{a+}^{\alpha}|g(b) h(b)| \\
& \quad+\left\|D_{b-}^{\alpha} g\right\|_{L_{1}([a, b])} I_{a+}^{\alpha}|f(b) h(b)| \\
& +\left\|D_{b-}^{\alpha} h\right\|_{L_{1}([a, b])} I_{a+}^{\alpha}|f(b) g(b)| .
\end{aligned}
$$

Proof. From (15) we have

$$
\begin{aligned}
& \mid 3 \int_{a}^{b} f(x) g(x) h(x) d x \\
& -\int_{a}^{b}[f(b) g(x) h(x)+f(x) g(b) h(x) \\
& +f(x) g(x) h(b)] d x \mid \\
& \leq \int_{a}^{b} \frac{|g(x) h(x)|}{\Gamma(\alpha)}\left(\int_{x}^{b}(t-x)^{\alpha-1}\left|D_{b-}^{\alpha} f(t) d t\right|\right) d x \\
& +\int_{a}^{b} \frac{|h(x) f(x)|}{\Gamma(\alpha)}\left(\int_{x}^{b}(t-x)^{\alpha-1}\left|D_{b-}^{\alpha} g(t) d t\right|\right) d x \\
& +\int_{a}^{b} \frac{|f(x) g(x)|}{\Gamma(\alpha)}\left(\int_{x}^{b}(t-x)^{\alpha-1}\left|D_{b-}^{\alpha} h(t) d t\right|\right) d x \\
& \leq \int_{a}^{b} \frac{|g(x) h(x)|}{\Gamma(\alpha)}(b-x)^{\alpha-1}\left(\int_{x}^{b}\left|D_{b-}^{\alpha} f(t) d t\right|\right) d x \\
& +\int_{a}^{b} \frac{|h(x) f(x)|}{\Gamma(\alpha)}(b-x)^{\alpha-1}\left(\int_{x}^{b}\left|D_{b-}^{\alpha} g(t) d t\right|\right) d x \\
& +\int_{a}^{b} \frac{|f(x) g(x)|}{\Gamma(\alpha)}(b-x)^{\alpha-1}\left(\int_{x}^{b}\left|D_{b-}^{\alpha} h(t) d t\right|\right) d x \\
& =\left\|D_{b-}^{\alpha} f\right\|_{L_{1}([a, b])} \int_{a}^{b} \frac{|g(x) h(x)|}{\Gamma(\alpha)}(b-x)^{\alpha-1} d x \\
& +\left\|D_{b-}^{\alpha} g\right\|_{L_{1}([a, b])} \int_{a}^{b} \frac{|h(x) f(x)|}{\Gamma(\alpha)}(b-x)^{\alpha-1} d x \\
& +\left\|D_{b-}^{\alpha} g\right\|_{L_{1}([a, b])} \int_{a}^{b} \frac{|f(x) g(x)|}{\Gamma(\alpha)}(b-x)^{\alpha-1} d x \\
& \leq\left\|D_{b-}^{\alpha} f\right\|_{L_{1}([a, b])} I_{a+}^{\alpha}|g(b) h(b)| \\
& +\left\|D_{b-}^{\alpha} g\right\|_{L_{1}([a, b])} I_{a+}^{\alpha}|f(b) h(b)| \\
& +\left\|D_{b-}^{\alpha} h\right\|_{L_{1}([a, b])} I_{a+}^{\alpha}|f(b) g(b)| .
\end{aligned}
$$

This proves the theorem.

Remark 10. If we take $h(t)=1, g(t)=1$, and hence $\left\|D_{b-}^{a} h\right\|_{\infty}=0,\left\|D_{b-}^{a} g\right\|_{\infty}=0$ in Theorem 9 , then we get Theorem 5.

Theorem 11. Let $p, q>1 ; 1 / p+1 / q=1, \alpha>1-1 / p, m=[\alpha]$, $f, g \in A C^{m}([a, b])$. Assume that $f^{k}(b)=g^{k}(b)=h^{k}(b)=0$, 
$k=1, \ldots, m-1$, and $D_{b-}^{\alpha} f, D_{b-}^{\alpha} g, D_{b-}^{\alpha} h \in L_{q}[a, b]$. Then

$$
\begin{aligned}
& \mid 3 \int_{a}^{b} f(x) g(x) h(x) d x \\
& -\int_{a}^{b}[f(b) g(x) h(x)+f(x) g(b) h(x) \\
& \quad+f(x) g(x) h(b)] d x \mid \\
& \leq \Gamma\left(\alpha+\frac{1}{p}\right)\left(\left\|D_{b-}^{\alpha} f\right\|_{L_{q}([a, b])} I_{a+}^{\alpha}|g(b) h(b)|\right. \\
& \quad+\left\|D_{b-}^{\alpha} g\right\|_{L_{q}([a, b])} I_{a+}^{\alpha}|f(b) h(b)| \\
& \left.+\left\|D_{b-}^{\alpha} h\right\|_{L_{q}([a, b])} I_{a+}^{\alpha}|f(b) g(b)|\right) .
\end{aligned}
$$

Proof. From (15) we have

$$
\begin{aligned}
& \mid 3 \int_{a}^{b} f(x) g(x) h(x) d x \\
& -\int_{a}^{b}[f(b) g(x) h(x)+f(x) g(b) h(x) \\
& \quad+f(x) g(x) h(b)] d x \mid \\
& \leq \int_{a}^{b} \frac{|g(x) h(x)|}{\Gamma(\alpha)}\left(\int_{x}^{b}(t-x)^{\alpha-1}\left|D_{b-}^{\alpha} f(t) d t\right|\right) d x \\
& \quad+\int_{a}^{b} \frac{|h(x) f(x)|}{\Gamma(\alpha)}\left(\int_{x}^{b}(t-x)^{\alpha-1}\left|D_{b-}^{\alpha} g(t) d t\right|\right) d x \\
& \quad+\int_{a}^{b} \frac{|f(x) g(x)|}{\Gamma(\alpha)}\left(\int_{x}^{b}(t-x)^{\alpha-1}\left|D_{b-}^{\alpha} h(t) d t\right|\right) d x .
\end{aligned}
$$

Applying Holder's inequality to (21), we get

$$
\begin{aligned}
& \mid 3 \int_{a}^{b} f(x) g(x) h(x) d x \\
& -\int_{a}^{b}[f(b) g(x) h(x)+f(x) g(b) h(x) \\
& \quad+f(x) g(x) h(b)] d x \mid \\
& \leq \int_{a}^{b} \frac{|g(x) h(x)|}{\Gamma(\alpha)}\left(\int_{x}^{b}(t-x)^{(\alpha-1) p} d t\right)^{1 / p} \\
& \quad \times\left(\int_{x}^{b}\left|D_{b-}^{\alpha} f(t) d t\right|^{q} d t\right)^{1 / q} d x \\
& \quad+\int_{a}^{b} \frac{|h(x) f(x)|}{\Gamma(\alpha)}\left(\int_{x}^{b}(t-x)^{(\alpha-1) p} d t\right)^{1 / p}
\end{aligned}
$$

$$
\begin{aligned}
& \times\left(\int_{x}^{b}\left|D_{b-}^{\alpha} g(t) d t\right|^{q} d t\right)^{1 / q} d x \\
& +\int_{a}^{b} \frac{|f(x) g(x)|}{\Gamma(\alpha)}\left(\int_{x}^{b}(t-x)^{(\alpha-1) p} d t\right)^{1 / p} \\
& \times\left(\int_{x}^{b}\left|D_{b-}^{\alpha} h(t) d t\right|^{q} d t\right)^{1 / q} d x \\
& =\frac{\left\|D_{b-}^{\alpha} f\right\|}{\Gamma(\alpha)} L_{q}([a, b]) \int_{a}^{b}|g(x) h(x)| \\
& \times\left(\int_{x}^{b}(t-x)^{(\alpha-1) p} d t\right)^{1 / p} \\
& +\frac{\left\|D_{b-}^{\alpha} g\right\|}{\Gamma(\alpha)} L_{q}([a, b]) \int_{a}^{b}|h(x) f(x)| \\
& \times\left(\int_{x}^{b}(t-x)^{(\alpha-1) p} d t\right)^{1 / p} \\
& +\frac{\left\|D_{b-}^{\alpha} h\right\|}{\Gamma(\alpha)} L_{q}([a, b]) \int_{a}^{b}|f(x) g(x)| \\
& \times\left(\int_{x}^{b}(t-x)^{(\alpha-1) p} d t\right)^{1 / p} .
\end{aligned}
$$

We have

$$
\left(\int_{x}^{b}(t-x)^{(\alpha-1) p} d t\right)^{1 / p}=\frac{(b-x)^{\alpha-1+1 / p}}{(p(\alpha-1)+1)^{1 / p}} .
$$

Substituting (22) into (21), we get the required inequality.

Remark 12. If we take $h(t)=1, g(t)=1$ and hence $\left\|D_{b-}^{a} h\right\|_{\infty}=0,\left\|D_{b-}^{a} g\right\|_{\infty}=0$ in Theorem 11 , then we get Theorem 6.

\section{References}

[1] B. G. Pachpatte, "On an inequality of Ostrowski type in three independent variables," Journal of Mathematical Analysis and Applications, vol. 249, no. 2, pp. 583-591, 2000.

[2] B. G. Pachpatte, "New Ostrowski and Grüss type inequalities," Analele Stintifice Ale Universitath Al.I Cuza Iasi, Tomul LI. s. I Mathematica, vol. 51, no. 2, pp. 377-386, 2005.

[3] G. A. Anastassiou, Intelligent Mathematics: Computational Analysis, Springer, Berlin, Germany, 2011.

[4] G. A. Anastassiou, "On right fractional calculus," Chaos, Solitons and Fractals, vol. 42, no. 1, pp. 365-376, 2009.

[5] E. A. Bohner, M. Bohner, and T. Matthews, "Time scales ostrowski and gruss type inequalities involving three functions," Dynamics and Systems Theory, vol. 12, no. 2, pp. 119-135, 2012.

[6] M. Bohner and T. Matthews, "Ostrowski inequalities on time scales," Journal of Inequalities in Pure and Applied Mathematics, vol. 9, no. 1, article 6, 8 pages, 2008. 
[7] G. A. Anastassiou, Fractional Differentiation Inequalities, Springer, Dordrecht, The Netherlands, 2010.

[8] I. Podlubny, Fractional Differential Equations, vol. 198, Academic Press, San Diego, Calif, USA, 1999.

[9] K. S. Miller and B. Ross, An Introduction to the Fractional Calculus and Fractional Differential Equations, John Wiley \& Sons, New York, NY, USA, 1993.

[10] G. A. Anastassiou, Advances in Fractional Inequalities, Springer, New York, NY, USA, 2011. 


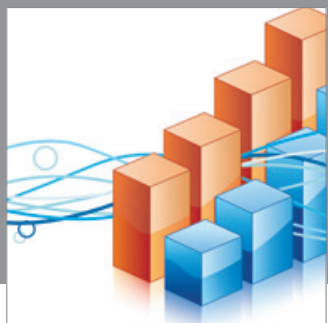

Advances in

Operations Research

mansans

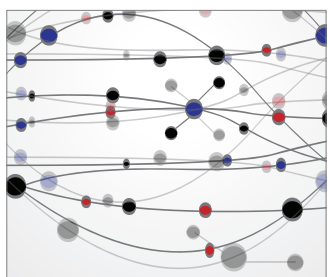

The Scientific World Journal
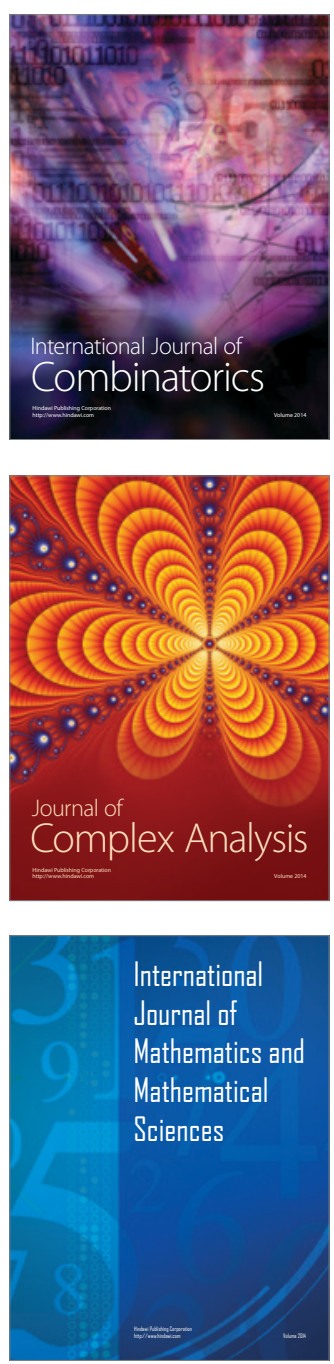
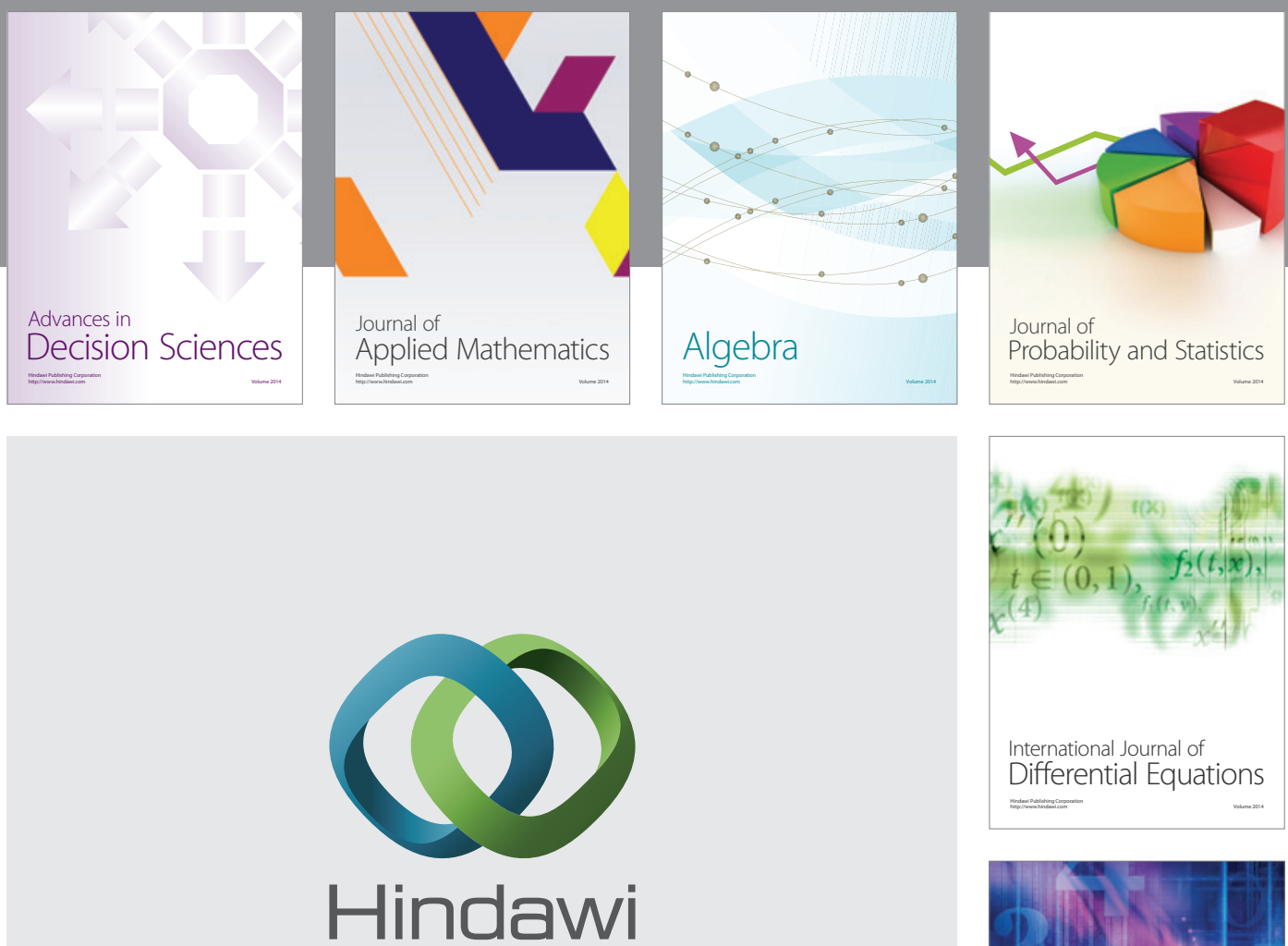

Submit your manuscripts at http://www.hindawi.com
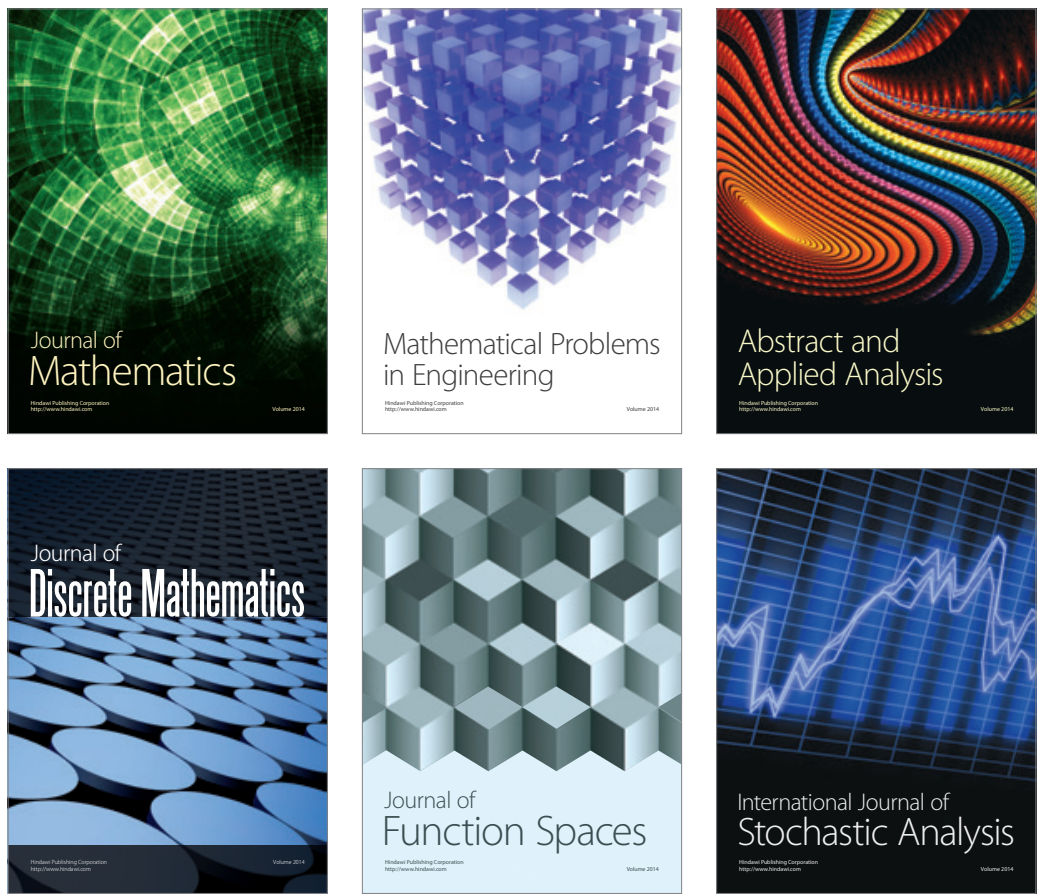

Journal of

Function Spaces

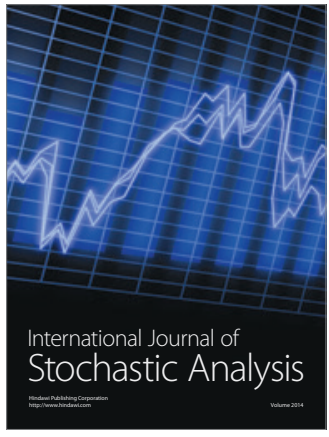

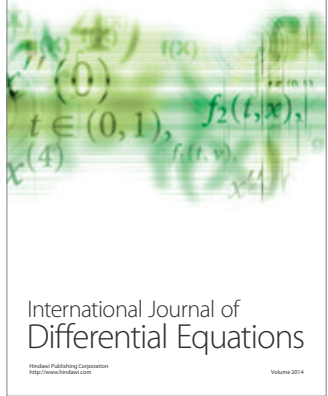
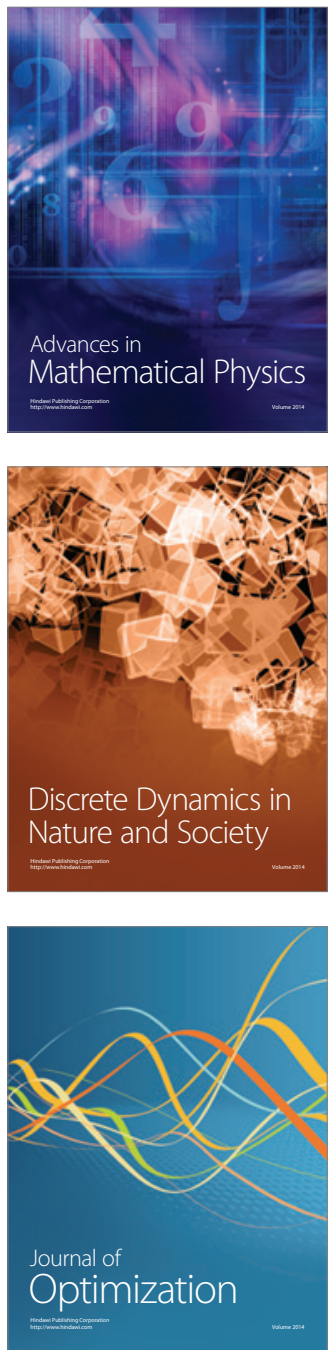